\title{
Coarse Segmentation of Textured Images Using Variance Analysis
}

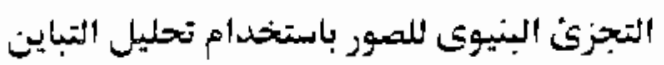

\section{Fatma M. Bayoumi² Samir I. Shaheen' Ayman A.M. Ibrahim ${ }^{2}$}

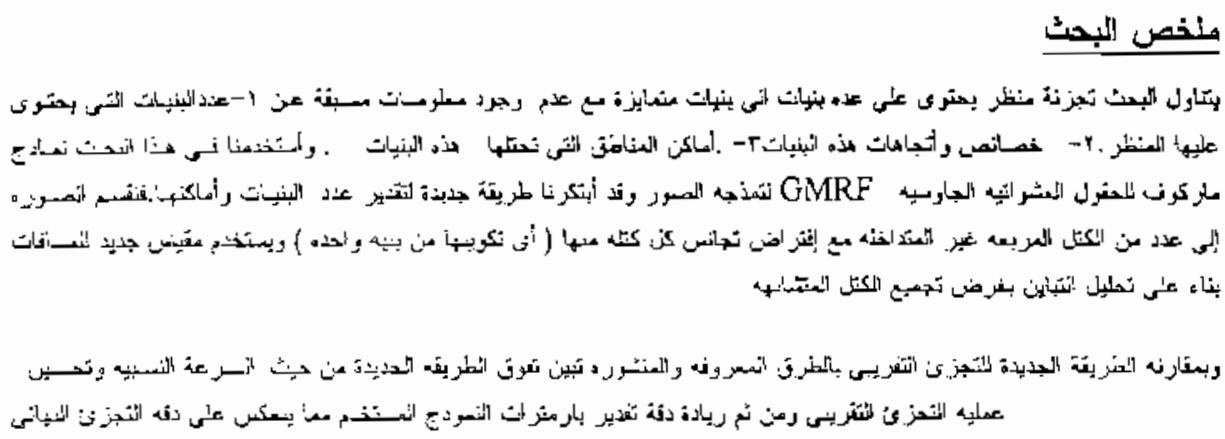

\section{ABSTRACT}

This paper presents a novel approach for the segmentation of a textured scene. The algorithm is inage-bosed, no specilic model is assumed for the image. Also, no a priori knowiledge abour the different texture regions, neither their number, nor their behavior is assumed. The algorithm partitions the image into small disjoint square windows, and the variance for each window data is caleulated. Then the $K$-means clustering algorithm is applied upon these windows. Together with the $K$-means algorithm, a new distance measure has been delined. This new distance measure was deduced from a stalistical test known as Bartlett's test based on the variance of the windows data. The same statistical test has aiso been applied but in a different fashion to determinc the number of diffcrent texrurcs in the image. The new image-based distance measure has been tested and compared to a model-based Euclidean distance measure, with each window modeled by a non-causal Gaussian Markov Rontom Field (GMRF). The results of the eomparison have shown that the new distance measurc is mueh simpler and faster, while yielding to a still robust and effective segmentation.

Accepted July 30, 1997

'Computer Engineerng Department. Fnculty of Enyineering, Caıro Universiry, Giza, Egypt.

E-Mail: SSHAHEENGERCU EUN EO

" Nationat Telecommunication Institule, 5 Mokjaaiam El-Düm Street, Naser Ciry, Cairo, Egypt.

C-Mail: BAYOMIANTI SCI EG

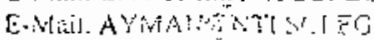




\section{Introciuction}

The objective of texture segmentation is to separate an image into differcnt ctgions of homogeneous behavior. It is at early processing task necessary to many applications such as: segnentation of scenes into distinct objects or reguons, classification or recognition of surface materials, computation of surface shapes and orientation, motion analysis, and image data compression.

Textures are generally defined according to one of two different aspects, narnelv structural and statistical. From the structural point of yiew, textures are formed by micro structures or grains, which are prinilives known as texels, and an accompanying grammar for the placement and reproduction of these texels. Obviously, this approach restrics to quitc regular patems, as $1 \mathrm{l}$ is highly difficult to deternine either the texels, or the granmar for non-regular textures. However, from the statistical point of view, texture is delined as a partern represented by a set of stalistical measurement features. Because of the random nature of natural texturs thisy resciurchers focused on the latter approach and applied the stochastic models for the image representarton. Special interest was ditected towards the Simulaneous Allto Regressive models $(S, H R)[\mathrm{I}-7]$ and the noncausal .Markov Random Field modtis (.WRF) [2-13]. Also, the Gibbs Disiribution (GD) received much attention [8].

This interese towards random field hodels encouraged many researchers to base their texture segmentation algorithms ypna !hese models. Derin and Ellion [14], and [15] made use of the Gibbs distribution, Whlle $[5,16-19]$ used the MRF models and $[5,20$, 21] exploired the SAR inodels.

To achieve good segmentation using the above models, the segmentation should be initiated with sufficient information about the number of textures in the image, and their model parameters. This problem can be solved if an algorithm is detined to coarsely segment tho inage (segmentation at a low resolution). Wilh a coarsely segmented image, we can detine the number of different textures in the image, their oceupied locations and ."trefore the. 'e sel parameters. Obvuusly, this algorithm should be simple and fast to initiate a fine segmentation algorithm with a minimized overhead.

Fitng et wi. [16] proposed an algorithm that performs coarse segnentation. Their algorithm is consiructed within the franework of a split and paradigm. It is based on the GMRF together with a likelihood ratiu test to guide the split and merge process. The algorithrn proposed by Fing ef al. leads to good results, but is too exhaustive to be used prior to a fine segmencer.

Since nany problems of image analysis are related to human visual perception, it will be quite useful to review a model of human texture pcrception. Texture research has been mostly driven by Ithesz' conjecture that the human visual system camot discriminate between textires differing only in third and higher-order statistics [22]. Although Gagalowicz [1] prescnted several counterexamples to the Julezs' conjecture, lie pointed-out that these counterexamples are very difficult to gcnerate, and that they are only faintly discriminable, and thus concluded rlual the Jute-s' conjecture seems to provide a useful bound for human visual texlure discrimunation. As a direct consequence 
of this conjecure, second-order statistics are frequently used as effective features for texture classification and discrimination. The second order moment (or simply the sample variance) is obviously one of the simplest second order statistics that can be used.

This paper presents a novel approach to coarsely segment a textured seene in an unsnpervised fashion. The algorithm starts by partitioning the image into a number of relatively snaall disjoint windows, and the variance is ealeulated for the window data. Becanse of their small size, most of the windows are homogeneous. We apply a wellknown vector quantization algorithm known as the $K$-means elustering algorithm upon the windows to cluster them in $K$ different classes (or textures). Together with the $K$ means algorithm, a new distance measure has been defined. This new distance measure was deduced from a stalistieal test known as Bartlett's test [23] based on the variance of the windows data. The same statisticai test has been applied but in a different fashion to determine the number of different clusters formed.

The algorithm results in a coarse segmented image, where the smoothness of the boundaries depends on the size of the used windows.

The coarse segmentation can be used in applications where it is required to estimare the texture locarions, which can also be used to model the textures according to some appropriate models. The algoritlun also provides a good initiation tor finer seginentation algorithms with a minimized overhead.

This paper involves(1)The coarse segmentation of textured scenes in an unsupervised environment, and (2)The definition of a new distance measure based on a statislical test to be used to discriminate between rextures.

The organization of the paper is as follows : Section II introduces the new distance measure. Section III defines the proposed segmentation algorithm. Section IV presents a comparison of the time eomplexity of the propused algorillum with other similar algorithms. Section V gives the segmentation and comparison results. Seetion VI is the conclusion with the recommended perspectives.

\section{The New Distance Measure}

To be useful, a distortion measure musl be tractable, so that it can be analyzed and computcd, and subjectively relevant, so that differences in distortion values can be visually discriminative. From [24], any distance function $d(x, y)$ can be accepted as having the qualitics of a distance measure if it satisfies the following rules:

$$
\begin{aligned}
& d(x, x)=0, \\
& d(x, y)>0 \quad \text { for } x \neq y, \\
& d(x, y)=d\left(y^{\prime}, x\right), \\
& d(x, y)+d(y, z) \geq d(x, z)
\end{aligned}
$$

Our new distance measure is applied on scalar data. It is based on the varianees of the compared data sets. It is okeu important to know with some degree of certainty whether the variarices of two data sers are the same, i.e. they do not differ significanlly; such variances are said to be homogeneous. We made usc of a statislical test known as Bartleft's test [23]. This test is applied to test the humogeneity of two or more variances. 
Bartletl's test is a special applicalion of the well-known $\chi^{2}$ test, in which we compare the difference between the total number of degrees of freedom times the natural logarithm of the pooled estimate of variauce and the sum, exiended over all samples. of the product of the degrees of freedom and the natural logarithm of the estimate of variance. Thus, if $n_{t}$ is the sampie size, $s_{i}^{2}$ is the estimate of variance from sample $i, k$ is the number of samples, and $\bar{s}^{2}$ is the pooled estimate of variance, then the Bartlent's lest requires the calculation of

$$
x^{2}=\frac{1}{c}\left[v \ln \overline{s^{2}}-\sum_{r=0}^{k-1}\left(v_{1} \ln s_{s}^{2}\right)\right]
$$

where

$$
\begin{aligned}
& s_{i}^{2}=\frac{1}{n_{i}-1} \sum_{0}^{n_{i}-1}\left(x-\tilde{x}_{i}\right)^{2} \quad \text { and } \bar{x}_{i}=\frac{1}{n_{i}} \sum_{0}^{n_{i}-1} x \\
& v=\sum_{i=0}^{k-1} v_{i} \quad \text { (4) }
\end{aligned}
$$

where $v_{i}$ is the number of degrees of freedom of sample $i\left(v_{i}=n_{i}-1\right)$

$$
\bar{s}^{2}=\frac{1}{v} \sum_{i=0}^{k-1} v_{t} s_{i}^{2}
$$

aud

$$
c=1+\frac{1}{3(k-1)}\left[\sum_{i=0}^{k-1}\left(\frac{1}{v}\right)-\frac{1}{v}\right]
$$

The distance measure we are proposing will be the $\chi^{2}$ test, calculated for two variances $(k=2)$ such that

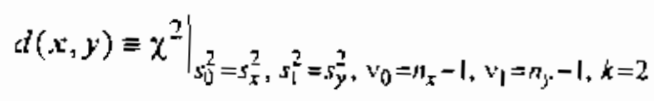

It can be ensily shown that this new distance measure complies well with the required properties in (1). 


\section{The Segmentation Procedure}

The proposed segmentation algorithm begins by partitioning the image into a number of relatively small disjoint witudows. The varianee for eaeh window data is calculated. Then, we apply the $K$-means algorithm, with the new distance measure defined in (7).

As previously diseussed, the proposed algorithm works in an unsupervised environment, in which the number of textures in the image is unknown. Therefore, when applying the $K$-means algorithm, we are faced with the problem of determining the number of clusters $K$ we want to be formed.

To determine the number of clusters (textures) in the image, we begin the segmentation process by assuming the image to be composed of only two textures, and apply the $K$-means algorithm. Then, we apply a statistical test based on the $\chi^{2}$ test detined in (2) to deternine whether the formed elusters are homogeneous or not to some pre-defined level of sig:ificance. If the clusters are found to be not homogeneous, we repeat the $K$-neans algorirhm while enabling it to form one more cluster. This procedure is repeated until we first find that two (or more) of the formed clusters are homogeneous. Findling that some of the formed clusters are homogeneous gives an indication that the number of formed clusters is greater than the actual number of textures in the image. Thus we can determine that the actual number of textures in the image is equal to the number of clusters formed at the previous stage.

\section{The Statistical Test Used to Guide the K-Means Algorithm}

\section{A. Determining The Homogeneity of Two Clusters}

To determine whether two ciusters $i$ and $j$ are homogeneous or not, we are going to test the homogeneity of their variances using the Bartleft's test by computing the $\chi^{2}$ measure defined in (2). We adopt the classical procedure for testing statistical hypotheses [23]:

1- Define the null hypothesis $H_{0}$ and the alternative hypothesis $H_{u}$

$H_{0}$ : the two variances are not significantly different (homogeneous).

$H_{\text {ad }}$ : the two variances are signifieantly different.

2- Calculate the lest statistie ( $\chi^{2}$ mensure) for the pre-detined number of degrees of freedom $(k=2$, then degrees of freedom $=1)$ 
3- Define the significance level $\alpha$ for the icst $\left(P\left[x^{2}>\chi_{\alpha}^{2}\right]=\alpha\right)$, and accordingly determine from the $\chi^{2}$ taoles the value $\chi_{\alpha}^{2}$ for the specified number of degrees of freedom (in our case, number of degrees of freedom $=1$ ).

The significance level $\alpha$ is a measure of the probaoility that the difference between the two variances is due to chance alone.

4- Perforn the test by comparing $\chi^{2}$ and $\chi_{\alpha}^{2}$.

If the caleulated value of $\chi^{2}$ exceeds $\chi_{\alpha}^{2}$, then, the probability that the difference between the two variances is due to ehance alone is even lower than the significance level $\alpha$, and we ate justified in rejecting the null hypothesis (and accepting the atiernate hypothcsis that the two variances are significantly different).

\section{B. The Proposed Algorithm}

1- Partition the image into a number of relatively sınall disjoint square windows, and calculare the variance for each window dara.

2- Inirialize the number of chisters to 2.

3- Calculate the chister centers.

4- Apply the $K$-means algoritlm using the distance measure defined in (7).

5- Test the homogeneity of the variances for the formcd clusters for a pre-defined significance level. If they are not significandly different go to 7.

6- Store the clustering results (windows classes), increment the current number of clusters by 1 and go to 3 .

7- Actual number of chisters = cument munher of chusters - 1, tetrieve the last clustering resulls.

8- Seginentation is done.

\section{Time Complexity and Storage of the Proposed Algorithm:}

In this section, we are going to compare our image-based distance messure to a model-based distance measure based on the Gaussian Markov Rundom Ficld Model (GMRF), from the point of view of the required computations and storage. We have chosen the GMRF model as it is extensively used in the recenl literature. The $4^{\text {th }}$ order GMRF has shown to be robust for the synthesis of natura! textures, and therefore we are going to use it in our comparison. windows.

As previously stated, the image is partitioned into smal! $M \times M$ disjoint square

Let $\Omega$ denote the sel of grid points of the $M \times M$ square window steh that:

$$
\Omega=\{s=(i, j): 0 \leq i, j \leq M-1\}
$$


and let $\{y(s)\}$ denote a random field with $y(s)$ the field at point $s$ representing the zeromean gray level.

Using the $4^{\text {th }}$ order GMLR, it is neaded to calculate for each window. a feature vector $\Phi=\left(\hat{\Theta}, \hat{\sigma}^{2}, \hat{\mu} \quad\right.$ where $\hat{\mu}$ is the sample mean, and $\hat{\Theta}$ and $\hat{\sigma}^{2}$ are respectively the GMRF parameters and the mean-square error and are defined as follows

$$
\text { Let } Q(s)=\operatorname{col}\left[y(s+r)+y(s-r), r \in N_{s}\right] \quad \text { and }
$$$$
N_{j}=\{(0,1),(1,0),(1,1),(-1,1),(0,2) \text {, }
$$$$
(2,0),(1,2),(2,1),(-1,2),(-2,1)\}
$$

then the leasi-square estimares $\hat{\Theta}$ of the parameters are defined as :

$$
\hat{\Theta}=\left[\sum_{i \in \Omega} Q(s) Q^{T}(s)\right]^{-1}\left[\sum_{s \in \Omega} Q(s) y(s)\right]
$$

and the meall apuare error $\sigma^{2}$ due to rhe model parameters is delined as

$$
\sigma^{2}=\frac{1}{M^{2}} \sum_{j \in \Omega}\left[y(s)-\hat{\Theta}^{T} Q(s)\right]^{2}
$$

\begin{tabular}{|c|c|c|}
\hline \multirow[t]{2}{*}{ Factor } & \multicolumn{2}{|c|}{$\begin{array}{l}\text { Computations Needed } \\
(m=\text { multiplication. } a=\text { division, } a=\text { addition })\end{array}$} \\
\hline & New Method & Traslitional Method \\
\hline $\begin{array}{l}\text { Features } \\
\text { Calculation }\end{array}$ & $W\left\{M^{2} a+M^{2} m+\mathrm{l} d\right\}$ & $\begin{array}{l}W\{(232 M+100) a \\
+(211 M+100) m+2 d \\
+10 \times 10 \text { matrix inversion }\}\end{array}$ \\
\hline$n$ & 0 & $W\{12 a+12 d\}$ \\
\hline K-neans & $\begin{array}{l}I\{3 a+4 d \\
+W K \text { (distance measure) } \\
=I\{3 a+4 d \\
\left.+W^{\prime}(3 a+2 n+1 a)\right\}\end{array}$ & $\begin{array}{l}I\{14 a+15 d \\
+W K(\text { distance measure })\} \\
=I\{14 a+15 d \\
+W K(19 a+10 m)\}\end{array}$ \\
\hline $\begin{array}{l}\text { Total } \\
\text { Com }\end{array}$ & $\begin{array}{l}\left\{\left(6 M^{2}+6\right) V\right. \\
+(27+19 J V K) \Omega a\end{array}$ & $\begin{array}{l}\left\{\left(1287,1 h^{2}+696+10 \times 10\right.\right. \\
\text { matrix inversion }) W \\
+(10++69 \mathrm{WK}) \cap a\end{array}$ \\
\hline
\end{tabular}

A. Required Computatiuns

Table 1. Computations needed to perform segmentation 
The proposed algorithm achieves a very large speedup over a model-based segmenter using a $4^{\text {th }}$ order GMRF. This speedup is due to several factors. These factors are the features caleulation, the fearures normalization, and the $\mathrm{K}$-means algorithm.

$$
\begin{aligned}
\text { segmentation time } & =\text { features calculation }+ \text { features normalization } \\
& +K \text {-means (using some distance measure) }
\end{aligned}
$$

If the image is partitioned into $V$ disjoint windows, and composed of $K$ lextures, then the computations needed for the segmentation are as shown in Table $L$. In the table, $I$ represents the number of iterations needed to perform $K$-means. The last row of the rable shows the total number of computations needed in terms of additions by eonsidering that typieally

Time of one muliplication $=$ at least Time of 5 additions

and Time of one division = ar least Time of 6 additions

speeditp $=\frac{\left(1287 M^{2}+696\right) W+(104+69 W K) I+10 \times 10 \text { matrix inversion }}{\left(6 W^{2}+6\right) W+(27+55 W K) I}$

For typical values of a $512 \times 512$ image, with 6 terrires, and choosing a $16 \times 16$ window', assuming the worst value of $\mathrm{I}=20$, the speedup is foumd to be $88.54 !$ And for a $256 \times 256$ image, with 4 termes, I $=10$, the speedtip is 144.56 !

\section{B. Required Storage}

For the proposed distance meusure, we only need to store the variance while for the GMRF, we need to store 12 parameters for each window.

\section{Results}

In this section. we present the segmentalion resulis performed on some images of real textures. All test images vere digitized into $64 \times 64$ santple images of 256 gray levels. The digirized inages have been used with no pre-processing. Eact image was subdivided into non-overlapping sindows. We used $16 \times 16$ and $8 \times 8$ windows. The sample variance was compuled for each window. Also, lhe $4^{\text {th }}$ order GMRF paramelers were caleulated, to help perform a comparison between the proposed distance measure and a model-based distance meusure.

The K-means algorithm was applied in both, the image-based and the modelbased cases. For the GMRF model, we applied the Euclidean distanee measure with normalized paralneters.

To determine the homogencity of the clusters formed by the $K$-means, we apply the statistical test defined in section IV.A. We used for this test a significance level $\alpha=$ $5 \%$. which equivalenty corresponds to $\chi^{2}$ values of $\chi_{\alpha}^{2}=3.841$. Thus if the computed value of $\chi^{2}$ for any two clusters is found to be larger than 3.84;, these two clusters ean be considered to be significantiy different. Figures I through 4 show the original image followed by the segmentation performed using the GMRF parameters and finally by that 
based on the proposed distance measure. The first row shows the experiments carried out using $16 \times 16$ windows, while the second row shows the experiments carried out using $8 \times$ 8 svindows.

The results have shown to be consistent for values of $\alpha$ ranging from $0.5 \%$ to $5 \%$. But if the textures are very similar a value of $\alpha=10 \%$ is more appropriate.

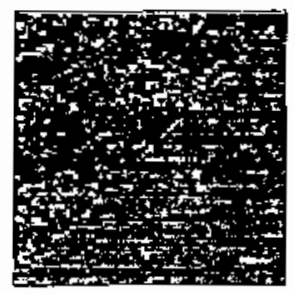

(a)

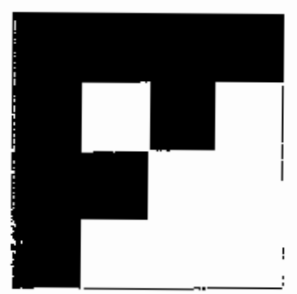

(b)

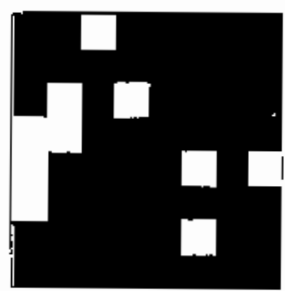

(d)

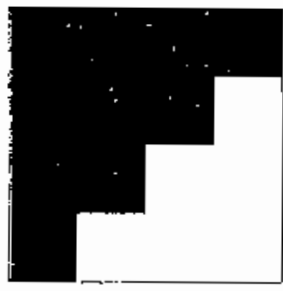

(c)

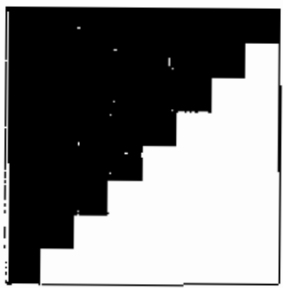

(e)

Fiğ. I. (a) Original image composed of "Flowers and Tiles" (b) Coarse seg̣. using GMRF, Euclidean distance, and K-means, $16 \times 16$ window (c) Coarse seg, using new algorthm, $16 \times 16$ window (d) Coarse seg. using GMRF, Euclidean distance, and K-means, $8 \times 8$ window

(c) Coarse seg, using new algorlthm, $8 \times 8$ windous

\section{Conclusion}

In this paper, we have presented a new algorithm for the coarse segmentation of a textured scene using an image-hased approach. The aigorithm works in an unsupervised fashion, with no required a priori knowledge about the number of textures in the image nor their behavior. The segmentation was performed using the $K$-meaths algorithm with a newvly defined distance measure. The algorithm was compared to algorithms using a model-based approach. The GMRF was used in the comparison. The results indicate that the proposed algorithm is nuch simpler and faster while using less storage and achieving

better segmentation. The new algorithm also gives good results even with small block

sizes (giving less coarse segmentation). The main problen that faces the proposed algorithm is the choice of the blocks sizes. If these blocks are chosen too large, regions of diffcring texlures may be lost. If the blocks are chosen too small, discriminating among similar textures may be difficult. Also, if the textures in the image are of significanlly. 


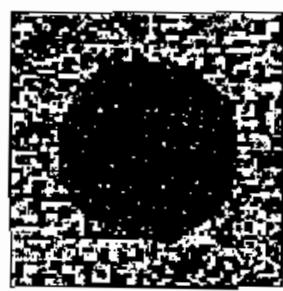

(a)

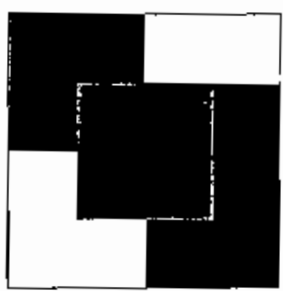

(b)

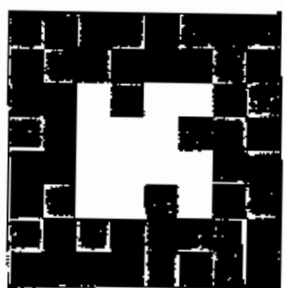

(d)

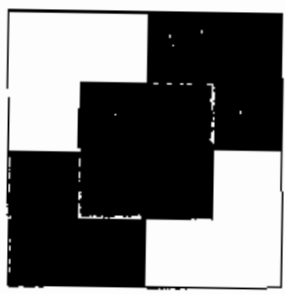

(c)

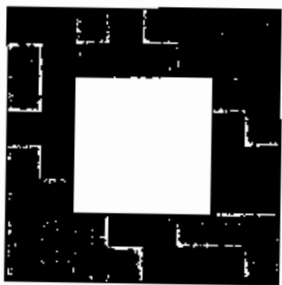

(e)

Fig. 2. (a) Original image composed of "Flowers, Water, and Sund" (b) Coarse seg. using GMRF, Euclidean distance, and $\mathrm{K}$-means, $16 \times 16$ window (c) Coarse seg. using sew algorillum, $16 \times 16$ window (d) Coarse seg. using GMRF, Euclidean distance, and K-means, BX8 window

(e) Coarse seg, using new algorithm. $8 \times 8$ window

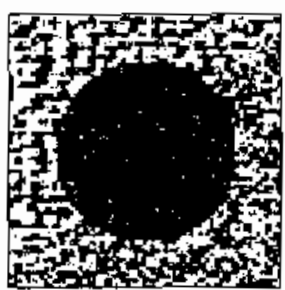

(a)

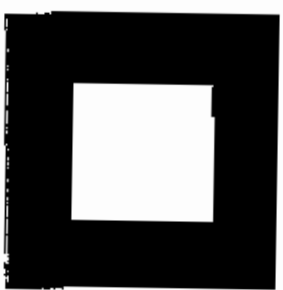

(b)

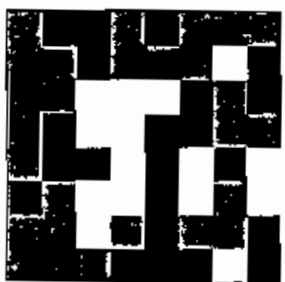

(d)

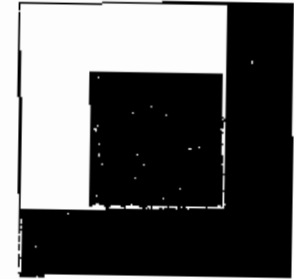

(c)

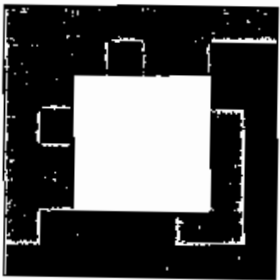

(e)

Fig. 3. (a) Orıginal inaye composed of "Raffia, Grass, and Rocks" (b) Coarse seg. using GMRF, Euclidean distance, and K-means, $16 \times 16$ window (c) Coarse seg, using new algorithm, $16 \times 16$ window (d) Coarse seg. using GMRF, Euclidean distance, and K-mears, $8 \times 8$ window

(e) Coarse seg, using new ajgorithm, $8 \times 8$ window 


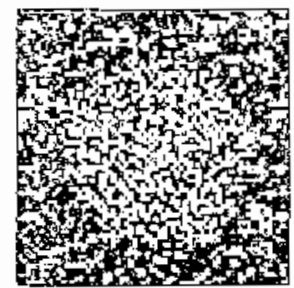

(a)

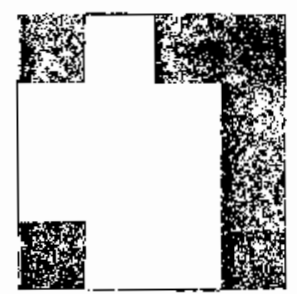

(b)

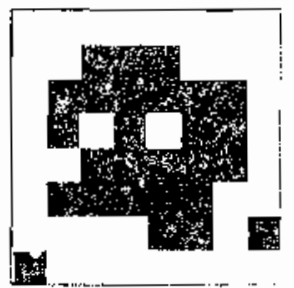

(d)

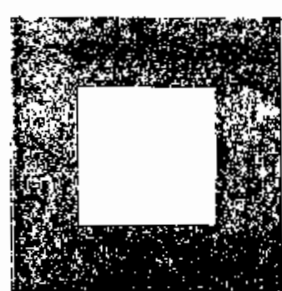

(c)

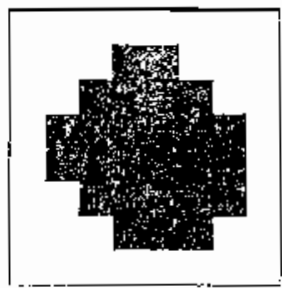

(c)

F:2 4. (a) Origilual inaye composed of "Rocks and Stucco" (b) Coarse seg. using GMRF, Euclidean لistance and $K-1$ treans, $16 \times 16$ window (c) Coarse seg, using new algon seg using GMRF, Euclidean distance, and K-rneans, $8 \times 8$ window

(e) Coirse seğ, using new algortlim. $8: 8$ window

different deglees of smoothness or coarseness, discrimination between them is a difficult task. There fore, some method should be applied to take mo account the grain size of the different textures

\section{REFERENCES}

[1] Pintr, W.K., Faugeras, OD., and Gagalowicz A., "Appilcálion of stochastle texture field models to Image processung", Proc. of the IEEE, vol. 69, no. 5, fp. 342-551, May 1981

[2] Jain, A.K, "Advances in malhematical modeis tor Inage processing", Proc. of the LEEE, vol. 69, no. 5. pp. 502.528, May 1931 .

[3] Kaslyup, R.L., "Randon field models on finite lanices for finite innages," Proc of the Annual Princeton Conti on intormation Science Systems, pp 215-220, 1981.

[-1] Kastıyap, R.L., and Chellappn, R., "Estimanon and choice ol neiglıbors in spatial-ınteraction models of "mages". IEEE Trans. on Intomation Theory, vol. IT-29. no. i, pp. 60-72, Jenuary 1933.

[5] Besag, J.E... "Spatial interacton and statisucal analysis of latuce systems", I of Roy Stat. Soc B, vol. 36. pp. 192-236, 1974.

[6] R.Azencen S.P Wang, and L Younes, "Texture Classification using windowed Fourier Fillers", lEEE. Voi PA.MI 19.No. 2, 1997.

[7] N Grordana and W Piecezynski, "Estuntation ol genertaized multisensor Hidden Markov Chains and Unsupervised image Segmentation", JFFE Vol, PANI 19., No. 5, 1997.

[8] Gemall. S., and Gemian. D. "Stchehastic relixation, Gibbs distribution, and the bayesıan restoration of

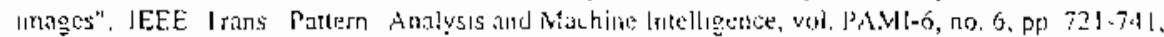
1934 
[9] Chellappa, R., and Kashyap, R.L., "Texture synthesis using spatial interation models", Proc. of IEEE Computer Soc. Conf. on Pritem Recognition and Image Processing, Las Vegas, pp. 226.230, June 1982 .

[i0] Besag, J.E., "On the statistical analysis of dirty picures", Joumal of Royal Statistical Society B, vot. 48. pp. 259-302, 1986.

[11] Chellappa, R., "Two-dimensional discreke Gaussian Markov Random Eield modeis tor image processıng", Progress in Parem Recognition 2, L. N. Kanal and A. Rosenfeld (Editors), Elsevier Science Publishers B. V. (North-Holland), pp. 79-1/2, 1985.

(12) Cross, G.R., and Jain, A.K, "Markov random field texture modeis", JEEE Trans. Pattem Analysis and Mochine Intelligence, vol. PAMl-5, no. !, pp. 25-39, January 1983 .

[13] Chellappa, R., Chanerjee, S. and Bagdazian, R. "Texture synthesis and compression using GaussianMarkov Random Field models", IEEE Trans. on Systens, Man, and Cyberm, vol. SMC-15, No. 2, pp. 298-303, March / April 1985.

[14] Derin, H., and Ellior, H., "Modeling and segmentation of noisy and rextured images using Gibbs randorn fields," IEEE Trans. Partem Analysis and Machine Intelligence, vol. PAMlI-9, no. I, pp. 39$55,1987$.

[15] Fung, P.W., Grebbin, G. and Attikiouzel, Y, "Model-based reglon growing segmentation of textured images", Proc. ICASSP, pp. $2313+2316,1990$.

[16] Laxshmanan, S. and Derin, D., "Simullaneous parameter eslimalton and segmentation of Gibbs random fields using simulaled annealing", IEEE Trans. Pattern Analysis and Machine Intelligence, vol. 11, pp. 799-813, Aug. 1989.

[17] Fnn, Z. and Cohen, F.S., "Unsupervised rexured image segmentation", Inremational . Conference on Intefligent Autonomous Systems, pp. 209-217,8-11 Dec. 1986, Antsierdanı.

[18] Cohen, F.S, and Cooper, D.B., "Simple parallel herarclical and relaxarion algorithms for segmentine non causal markovian randarn fields", IEEE Trans. Pattem Analysis and Machine fntelligence, vol. PAM!-9, no. 2, pp. 195-219, 1987.

[19] Geman, S., and Graffigne, C., "Markov random fieid image models and Iheir applications to computer vision", Proc. of the lnt. Congress of Mathematicians, Berkeley, USA, PP. 1496-1317. 1986.

[20] Cheilappa, R., Chatterjee. S., and Bagdazian, R., "Texture synthesis and compression using GaussianMarkov Random Fiejd models", IEEE Trans. on Systems, Man, and Cybem, yol. SMIC-15, No. 2, pp. 298-303, March/April 1985.

[2!] Khoranzad, A., and Chen, J., "Unsupervised segmenlation of textured images by edge detection in multidimensiona! 「eatures", IEEE Trans. Pałtern Analysis and Machine Incellixence, vol. PAMl-1!, 110. 4, pp. $414-421$, April 1989.

[22] Berry, J. R. Jr. and Goutsias, J., "A comparative study of matrix measures for maximum likelihood iexrure classificaton", IEEE Truns. Systems, Man. and Cyben., vol. 21, no. 1. pp. 252-261, 1991.

[23] Neville. A.M., Kennedy, J.B., "Basic Statistical Methods for Engineers and Scientssts", Intematiunal textbook company, 1970.

[24] Misisel, W.S., "Computer-Oriented Approaches ta Pattern Recogntton", Academic Press, 1972 